\title{
A NEW STRATEGY FOR MANAGEMENT AND RECONFIGURATION OF SELF-CONTAINED POWER SYSTEMS
}

By:

K.R. Davey

R.E. Hebner

IEEE Transactions on Power Delivery (under review)

PR 383

Center for Electromechanics

The University of Texas at Austin

PRC, Mail Code R7000

Austin, TX 78712

(512) $471-4496$

$07 / 11 / 2006$ 


\title{
A New Strategy for Management and Reconfiguration of Self-Contained Power Systems
}

\author{
Kent Davey, Fellow, IEEE and Robert Hebner, Fellow, IEEE
}

\begin{abstract}
Power systems on naval vessels and airplanes are good examples of self-contained power systems. These types of systems are useful for testing reconfiguration, particularly ones that might be implemented continuously, not just under compromised conditions. Determining the best operating condition in real time is challenging, since it discourages stochastic approaches. A fixed grid representation of the dynamic loads is recommended, employing a phasor algorithm to update the load impedances. A new subspace approach for solving the reconfiguration is presented, and compared to branch and bound algorithms. Reconfiguration is studied for a test system with $\mathbf{1 6}$ million switch options. Also discussed is how this information can be used in the design of the power grid a priori.
\end{abstract}

Key Words - integer optimization, phase analysis, stochastic

\section{INTRODUCTION}

As the Navy begins to exploit the advantages of the all-electric ship, the approach to power management is evolving. Amy[1] points out that when electric loads were small, power management was done through hard-wired controls, relay logic, and vital/non-vital distinctions in load aggregation. In this approach, load sharing was largely determined by those who designed or modified the ship.

As the electric loads become larger, the power management philosophy is shifting to an approach of "budgeted sharing." In this approach, the available generating capacity is allocated to loads. As long as the power actually used is well below the budgeted power, the system is adequate. As the power needed approaches the budgeted power level, loads must be shed or new capability brought on line. This is a step closer to an automated approach to power control.

This paper describes research toward a more complete and highly functional power management philosophy. The underlying logic of the system is to achieve continuous optimization of the power system. During routine operation, the system could, for example, reconfigure itself occasionally to minimize losses as the loads change. If one or more components fail, due to natural or external forces, the system is capable of reconfiguring itself to provide power to as many of the loads as are still functional. If the functioning loads exceed system capacity, the priority loads will need to be served. The approach is to have the system optimization occur within several milliseconds of any significant change to achieve minimum disruption. After sufficient time for a human to assess the situation, the operator can modify the optimization criteria to achieve different functionality, if

Both K. Davey and R. Hebner are with the Center for Electromechanics, University of Texas, 10100 Burnet Rd, EmE 133, Austin, TX 78758, (email k.davey@mail.utexas.edu,r.hebner@mail.utexas.edu). necessary. This approach melds the heretofore separate functions of protection, control, load flow analysis, and operational efficiency.

The goal of reconfiguration varies somewhat in land based systems. For most unit commitment studies, the objective is to minimize cost while delivering demand power subject to the constraint that no transmission line ratings be exceeded. The constraints are frequently added as penalty functions on the cost [2] or as Lagrange multipliers on the cost function [3]. The optimization index in this paper is the difference between power delivered and power lost in transmission, subject to the constraint that no line or generator load capability be exceeded. The equivalent trunk line impedances are used exclusively for this analysis. Discrete variable optimization is favored for global solutions; it is only practical when the system size is limited. Integer-based programming optimization is faster, but rarely finds the global configuration minimum.

\section{Motivation}

The following questions motivated the research for this paper:

1. Should the reconfiguration system run only under attack or compromised conditions?

a. Why not run it all the time and rename it a power management system?

2. Should the near real time reconfiguration on a transient solution of Kirchoff's circuit laws?

a. Why not base it only on a simpler equivalent impedance representation of the system? Only the impedance values, not the equations change with time. An optimization algorithm has a better chance of running in real time.

b. Naval ship systems have unique qualities, not the least of which are very high power intermittent loads such as rail guns and active armor. Fig. 1 shows an example of the grid system in view. Multiple loads are tapped off each trunk line. The parallel impedances, $Z_{p}$, represent the sum of all such tapped loads in a trunk line, while the series loads, $Z_{\mathrm{s}}$, represent transmission impedance. An intelligent reconfiguration system should have the ability to maximize power delivery, while minimizing loss dynamically.

3. If we adopt the equivalent impedance avenue, is the processing time required to convert voltage and current measurements to equivalent impedances fast enough to make a difference? Will it be accurate enough to make meaningful recommendations? 
a. An analytic solution combined with parallelization has proved that this step can now be performed in less than 2 us [4].

4. If we adopt the view that this management system operate continuously, what is the optimization index and constraint?

a. Loss power - less useful power

5. How should the optimization be performed?

a. Is it enough to find the nearest working solution?

b. If the algorithm works effectively, can it be used as an inverse algorithm to advise trunk line current carrying capabilities?

c. Stochastic algorithms are classically slow.

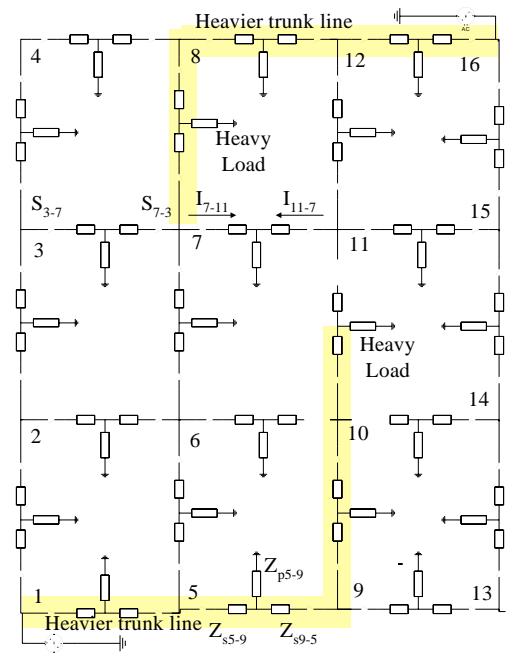

Fig. 1 Example of generic grid layout which lends itself to multi-load line use.

\section{MODELING GUIDELINES}

The following theses are posited for a self-contained power system such as that on a shipboard system:

1. A near real time data stream of voltage and current can be translated in about one-fifth of a cycle to magnitude and phase information.

2. Magnitude and phase information allows trunk lines to be replaced by their equivalent series and parallel impedance.

3. Load control can be implemented based on equivalent circuit representation treating the trunk lines as discrete global units.

4. Resource allocation and smart distribution is realized by considering the grid an integrated system, not one of isolated feeders, each free to carry partial or full power to all loads.

5. Each trunk line has a current rating. Continuous, not simply fault based, system re-configuration is the norm for this system. The optimization problem is to minimize parasitic transmission loss subject to the current ratings of each trunk segment. Large trunk segments designed for special purposes, e.g. electromagnetic launchers and railguns, should be available for other tasks when off duty to minimize parasitic system loss.
This paper focuses on items 45 .

\section{SYSTEM RECONFIGURATION}

In its simplest form, reconfiguration is about selecting switch settings to maximize power delivery while respecting load line capability. Generator loss and compromised system components and lines complicate this decision. More sophisticated objectives might add the criteria of minimizing transmission loss. If $R_{s}$ represents the series resistance components, $Z_{p}$ the load impedances, $I_{s}$ the series current through the lines, and $I_{p}$ the current through the loads, this criteria could be expressed mathematically as

$$
\begin{aligned}
& \Im=\sum I_{s}{ }^{2} R_{s}-\sum I_{p}{ }^{2} Z_{p} \\
& \text { subj } \\
& I_{s} \leq I_{\text {trunk Rating }} \\
& \text { Gen }_{\text {Load }} \leq P_{\text {Rated }}
\end{aligned}
$$

The last constraint ensures that no generator exceeds its rating. When loads have different weighting priorities $\mathrm{w}_{\mathrm{j}}$, the index changes to

$$
\mathfrak{s}=\sum I_{s}{ }^{2} R_{s}-\sum_{j=1}^{m} w_{j} I_{p j}{ }^{2} Z_{p j}
$$

subj

$I_{s} \leq I_{\text {trunk Rating }}$

Gen $_{\text {Load }} \leq P_{\text {Rated }}$

A binary integer programming problem takes the form

Minimize $\vec{f}^{\prime} \vec{x}$ such that $\mathbf{A} \vec{x} \leq \vec{b}$

The unknown $\mathrm{x}$ can only have the value 0 or 1 . Branch and bound algorithms have been found especially powerful for these types of problems [5] [6] [7]. The algorithm proceeds by treating the variables $\mathrm{x}$ as continuous. Suppose a solution of 0.45 is delivered for $x_{1}$. Two new problems are then generated (referred to as a branch); the first will have the added constraint $\mathrm{x}_{1}=0$, while the second branch will have the constraint $x_{1}=1$. The branches result in a decision tree with $2^{n}$ possibilities at the bottom of the tree as shown in Fig. 2. The algorithm has to decide whether to branch or to jump to the next node which is the opposite setting for the existing switch. If the relaxation problem returns an infeasible solution or its optimization index is greater than the current best integer value, that node is removed, and no other branches are searched below that node. If a better integer solution is found than the best existing integer solution, it updates the current best solution and moves on to the next node. Lastly, if an objective is found to be better than the current best value, but the solution is not integer, than the algorithm branches and proceeds down the tree. 


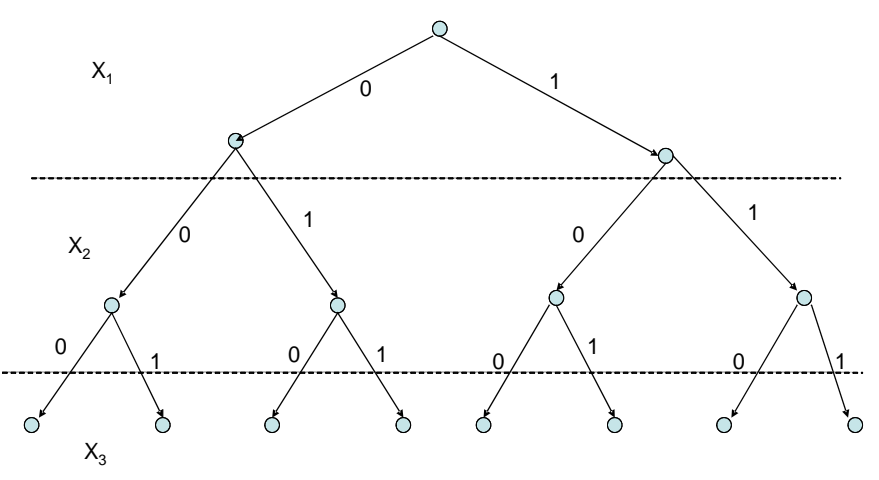

Fig. 2 Branching used in the combinatorial integer programming algorithm proposed for reconfiguration.

Branching is what causes the problem to grow. The key to this algorithm is that a branch is not formed unless the performance index is shown to improve at a node when the unknown is a non-integer value.

This algorithm can be performed with great speed if the problem can be linearized about some operating point. The procedure is as follows.

1. Assume a switch configuration $\mathrm{x} 0$, even one that is not feasible is suitable to start. $\mathrm{X}$ is a binary vector having value 0 or 1 ; each component of the vector represents a switch.

2. Compute the optimization index $\mathfrak{I}_{0}$ in (1) and the commensurate line currents $\mathrm{I}_{\mathrm{s} 0 \text {. }}$

3. As each switch is toggled sequentially, compute the change in the optimization index $\Delta \widetilde{S}$ and the line currents $\Delta \mathrm{I}$. The objective is to derive an approximate expression for the current change and index change at the present state. Let $\vec{I}$ represent a vector of all the currents of interest. The representation sought is

$\vec{I}=\vec{I}_{0}+\left[\begin{array}{cccc}\frac{\partial I_{1}}{\partial x_{1}} & \frac{\partial I_{1}}{\partial x_{2}} & \cdots & \frac{\partial I_{1}}{\partial x_{n}} \\ \frac{\partial I_{2}}{\partial x_{1}} & \frac{\partial I_{2}}{\partial x_{2}} & \cdots & \frac{\partial I_{2}}{\partial x_{n}} \\ \vdots & \vdots & \cdots & \vdots \\ \frac{\partial I_{m}}{\partial x_{1}} & \frac{\partial I_{m}}{\partial x_{2}} & \cdots & \frac{\partial I_{m}}{\partial x_{n}}\end{array}\right]\left[\begin{array}{c}x_{1} \\ x_{2} \\ \vdots \\ x_{n}\end{array}\right] \equiv \vec{I}_{0}+\mathbf{A} \vec{x}$

Every switch setting change delivers information about one column of the matrix in (4). By tracking the changes in the optimization index, the vector $\vec{f}$ is computed

$\mathfrak{\Im}=\mathfrak{\Im}_{0}+\left[\begin{array}{llll}\frac{\partial \mathfrak{I}}{\partial x_{1}} & \frac{\partial \mathfrak{I}}{\partial x_{2}} & \cdots & \frac{\partial \mathfrak{I}}{\partial x_{m}}\end{array}\right]\left[\begin{array}{c}x_{1} \\ x_{2} \\ \vdots \\ x_{n}\end{array}\right] \equiv \mathfrak{\Im}_{0}+\vec{f} \cdot \vec{x}$
$\vec{b}=\vec{I}_{S-\text { rated }}-\vec{I}_{0}$

\section{A. A Reconfiguration Test Problem}

Consider the three by three test grid shown in Fig. 3. Work the problem with quantities expressed in per unit. Let $\mathrm{V}_{1}=\mathrm{V}_{2}=1$, $Z_{s}=0.1$, and $Z_{p}=1$. Listed adjacent to the arrows is the value of current determined by solving equations Kirchoff's voltage laws for the circuit. When the switches are closed, the resulting load currents are those displayed.

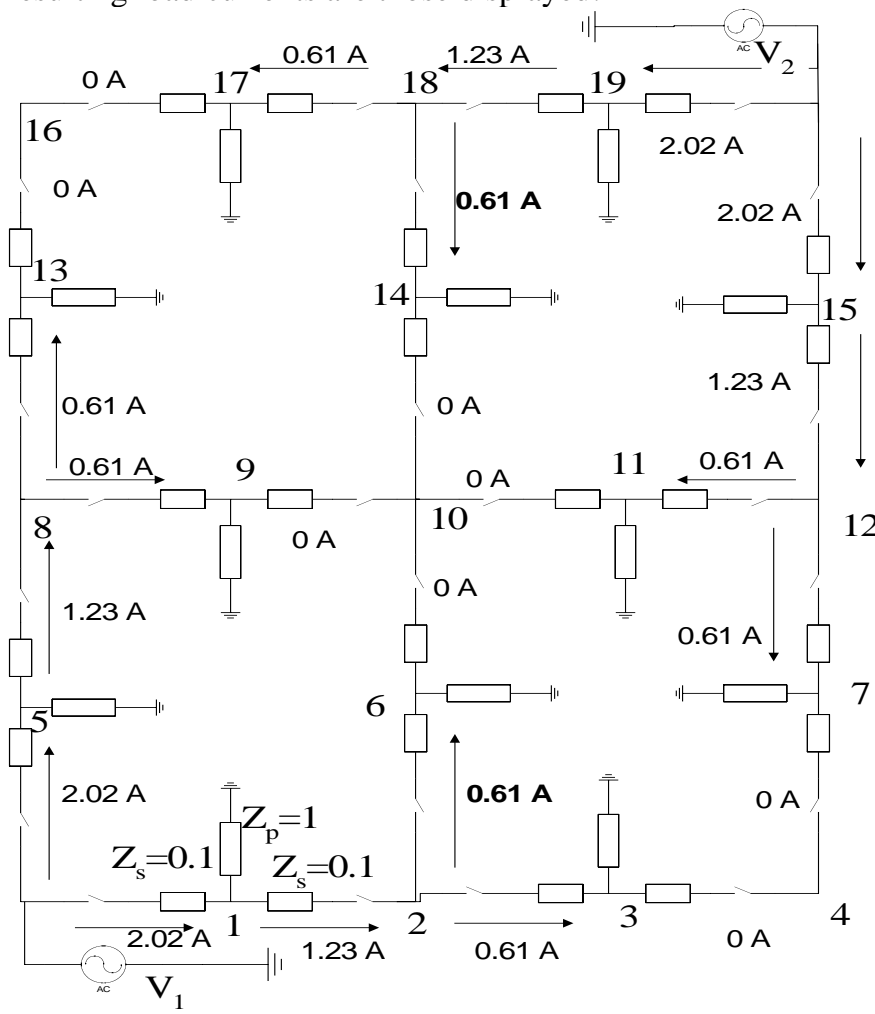

Fig. 3 Test grid for reconfiguration analysis.

Lowering the maximum current running on the trunk line between the source $\mathrm{V}_{1}$ and node 2 to $2 \mathrm{~A}$ may appear to be a minor perturbation. Fig. 4 shows the optimum constraint with the additional proviso that no trunk line be without power, determined by an exhaustive search of the variable space. Since per unit values are employed, the current through every parallel load should be in the range of 0.7 to 0.9 A for a $1 \mathrm{pu}$ load. Optimal configurations for tightly coupled systems are not intuitive. The optimization index (1) when only switch 1 or switch 2 on trunk line 1 is switched is -1.36 and -2.41 respectively, whereas with the optimal configuration shown, the index is -2.85 . The point to be noted is that a considerable improvement in power quality with loss reduction can be realized by performing some sort of optimization.

The vector $b$ in (3) is 


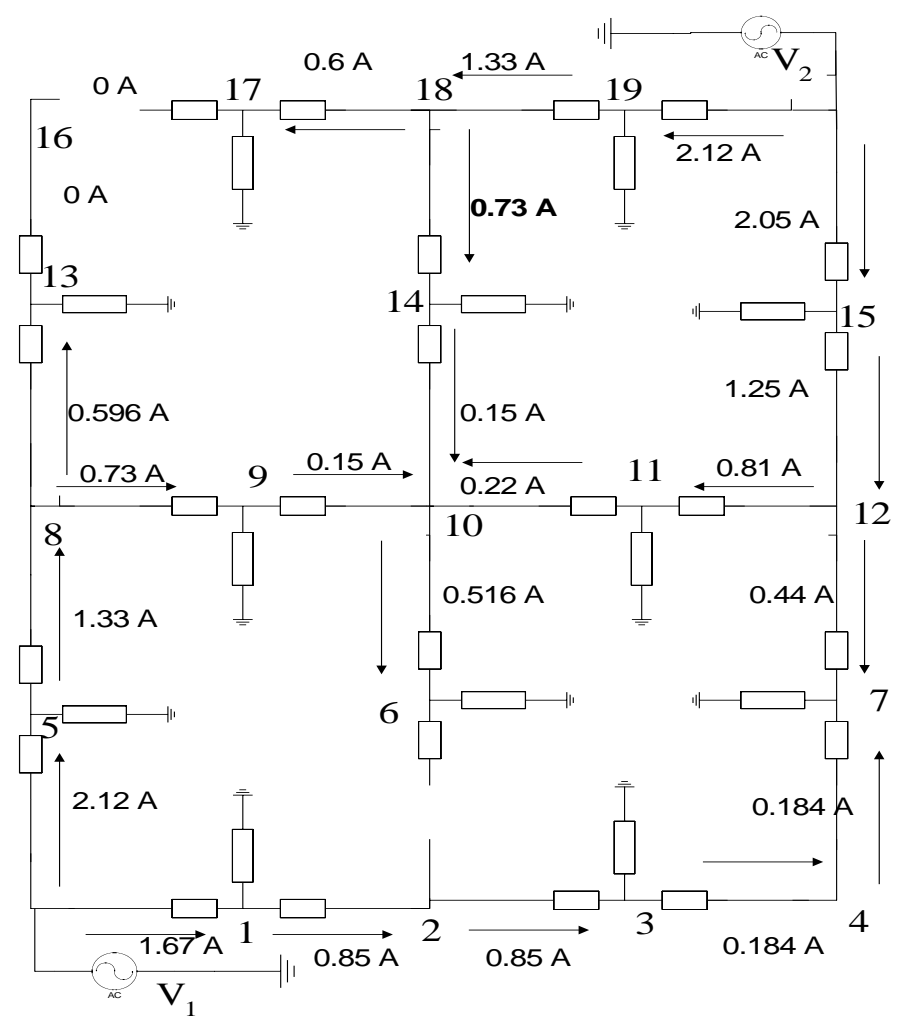

Fig. 4 New configuration resulting from the requirement that trunk line \#1 carry no more than $2 \mathrm{~A}$.

Is it necessary to add the constraint that power be delivered to every parallel load? Assume trunk line 1 between the voltage source and node 2 is constrained to carry no more than $2 \mathrm{~A}$ as above, but that no requirement exists for power delivery to every trunk line. With no power delivery constraint to every trunk line, the new configuration becomes that shown in Fig. 5. The point of the exercise is to confirm that the constraints are required.

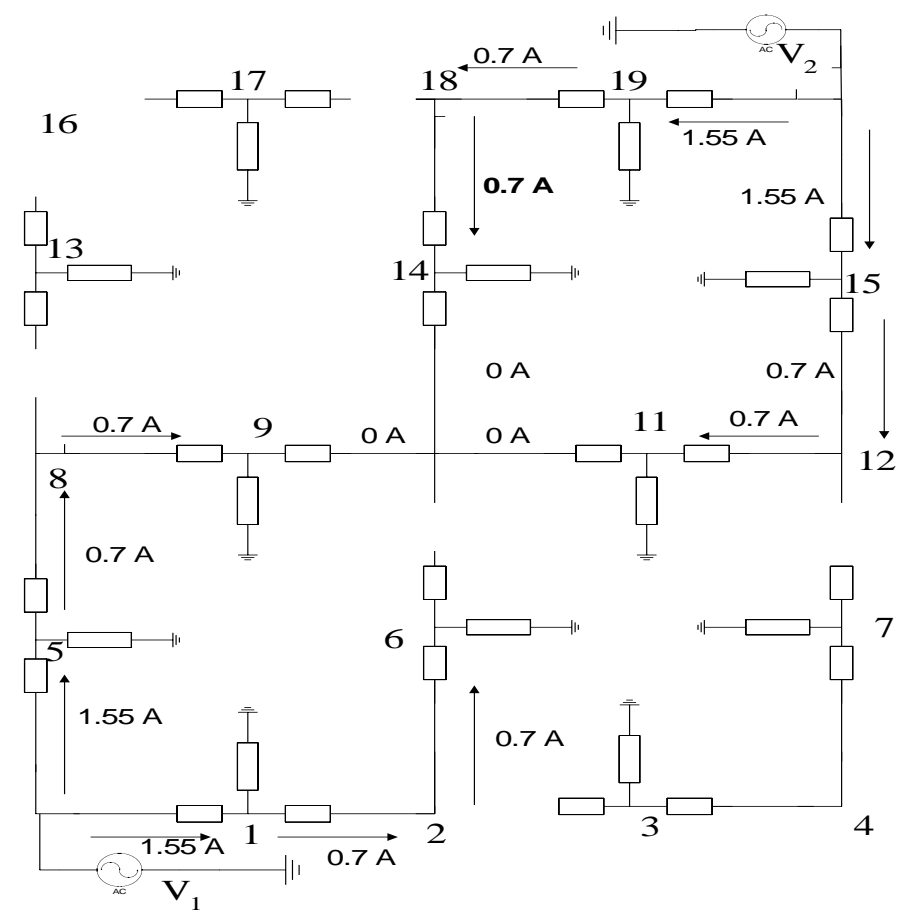

Fig. 5 Configuration resulting from constraining the current between $\mathrm{V}_{1}$ and node 2 to be less than $2 \mathrm{~A}$, with no constraints that power be delivered to every trunk line.

\section{B. Binary Algorithm Performance}

The recommended algorithm works exceedingly well, being both fast and accurate. Although this system appears simple, there are 16,777,216 possibilities; even when constraints are added to exclude all configurations excluding power from loads, the number of possibilities is 217,728 . Like all nonstochastic optimization algorithms, this one depends on the starting condition. There are numerous local minima throughout the solution space. The system can operate in these other configurations, and all the constraints are satisfied. The generators and transmission lines will operate at these local minima at under-rated conditions, but the system will not be at optimum performance. Local wells force the use of Monte Carlo starting techniques. Because of the algorithm's efficiency in building only a small sub-set of the solution tree, this procedure is tolerable. 

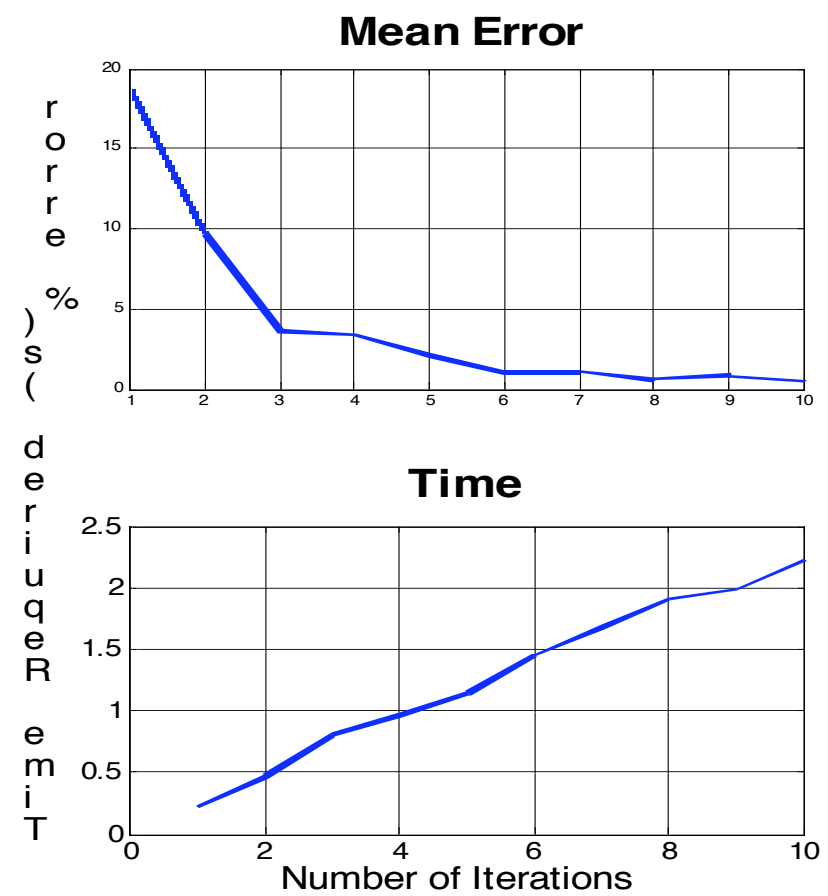

Fig. 6 Performance of the binary integer optimization algorithm.

Fig. 6 shows the performance delivered by the algorithm. A random switch configuration is chosen and the algorithm is started from this position, and followed to the optimal position; this sometimes requires a restart if no feasible solution can be found from the starting position. The matrices in (4) and (5) must be recomputed for each restart. A Monte Carlo computation involves marking the optimization index for a feasible solution and repeating the problem a number of times, searching for a new optimization within the Monte Carlo loop. The time continues to increase almost linearly with the number of Monte Carlo iterations; the departure from linearity depends on the speed with which the integer programming proceeds, i.e., how far the algorithm must proceed down the tree. The results would suggest that at least 6-8 iterations be employed to ensure an error near $2 \%$, quite acceptable for the relatively short time required. Because of the strategy chosen for branch decision, this algorithm is recommended for large scale systems.

\section{DISCUSSION}

1. A reconfiguration algorithm that uses continuously updated impedance from voltage and current measurements will incorrectly configure the system to low impedance cold loads.

The working assumption inherent to this objection is that the reconfiguration is not running continuously. As computer speed and algorithm efficiency increase, this assumption is unwarranted, and reconfiguring the system through a transient might not only be possible, but advantageous. Stability on these tightly coupled systems is unlikely to be affected. The obvious method of dealing with the objection if the reconfiguration requires multiple cycles is to place a delay in the system to overcome the transient.

2. This system is binary; the configuration switches are either open or closed. Why not use stochastic methods such as genetic and simulated annealing?

This team has investigated both these techniques for this problem [8]. These techniques work, but they are classically slower than other direct methods. Further attention will be given to a genetic algorithm tailored to binary input.

3. The numerical size of the system proposed grows as $2^{\mathrm{n}}$, where $\mathrm{n}$ is the number of switches. Real time reconfiguration for large systems is hopefully bankrupt.

Although the objection has foundation, there are two considerations. First, the placement of the switches is open; a large number of tapped loads can be placed on a trunk line. Second, another approach under investigation shows promise. In this subspace approach, only a smaller subset of the total switches is considered. The solution toggles between the smaller active subset of active switches, i.e., the solution is sought with switches 1-9 active, then 10-18, then 1-9, etc. An exhaustive search for the best configuration is sought for each subset. Convergence is usually witnessed in 2-3 iterations. It finds a solution greater than $90 \%$ of the time and is usually within $10 \%$ of the global index.

\section{CONCLUSIONS}

A special discrete parameter optimization program is run dynamically to determine the trunk-line switch settings commensurate with greatest power deliver and smallest parasitic loss. Steady state reconfiguration is effectively accomplished by branch and bound linear programming techniques. The salient points are as follows:

1. Treat the system as a number of feeder trunk lines each with its own equivalent ' $T$ ' impedance.

2. Continuously monitor current and voltage on either end of a trunk line with the equivalent impedances computed continuously from the voltage and current measurements.

3. Use a binary branch and bound integer programming optimization to determine the best configuration of switches to maximize power delivery and minimize losses in steady state operation.

Using reconfiguration algorithms in an advisory capacity looks promising, and will be the subject of a future paper. Consider sequentially simulating damaged lines throughout the grid; for each case, the reconfiguration algorithm is run with the constraint on minimum power delivery for weighted loads, maximizing the index in (1). The maximum of the 
current ratings obtained for all cases represents a design choice for the construction stage. Such a choice guarantees that the system reconfigure and manage the loss of any one line without compromising system performance. Comparable exercises could be performed for more extensive damage scenarios.

\section{REFERENCES}

[1] John Amy "Ship Integration of High-Power Weapon Systems for Electric Warships," American Society of Naval Engineers Conference: High Powered Weapon Systems for Electric Ship 2004, Annapolis, MD, USA, Dec. 2004.

[2] John J. Shaw, "A direct method for security constrained unit commitment," IEEE Trans. Power Systems, vol. 10, no. 3, August, 1995, pp. 1329-1342.

[3] C.L. Tseng, X. Guan, and A.V. Svoboda, "Multi-area unit commitment for large scale power systems," IEE Proc. Gener. Transm. Distri., vol. 145, no. 4, July 1998, pp. 415-421.

[4] Kent Davey, "Fault Analysis on a Naval Power Grid using Equivalent Impedances," IASME Transactions, Issue 2, vol. 1, April 2004, pp. 247-252.

[5] Wolsey, Laurence A., Integer Programming, John Wiley \& Sons, 1998.

[6] Nemhauser, George L. and Laurence A. Wolsey, Integer and Combinatorial Optimization, John Wiley \& Sons, 1988.

[7] Hillier, Frederick S. and Lieberman Gerald J., Introduction to Operations Research, McGraw-Hill, 2001.

[8] C. Meek, K. Davey, D. Tucker, B. Shutt, and R. Hebner, "Approaches to Shipboard Power Management," ASNE Reconfiguration and Survivability Symposium 2005, Jacksonville FL, Feb. 16-17, 2005. 